\title{
High-Resolution Broadly-Tunable MOPA-Based Terahertz Spectrometer to Non-Destructively Probe and Modulate Protein Electrodynamics
}

\author{
Richard J. Lewis ${ }^{a}$, Ee-Leong Lim ${ }^{a}$, David J. Rowe ${ }^{a}$, Shaif-ul Alam ${ }^{a}$, Johan Nilsson ${ }^{a}$, Chan-Wook Baik ${ }^{b}$ \\ Ogan Gurel ${ }^{b, c}$, James S. Wilkinson ${ }^{a}$, David J. Richardson ${ }^{a}$ \\ a Optoelectronics Research Centre, University of Southampton, Southampton, SO17 1BJ UK \\ ${ }^{b}$ Samsung Advanced Institute of Technology, Yongin 446-712, Korea \\ ${ }^{\circ}$ Samsung Advanced Institute for Health Sciences \& Technology, Seoul 135-710, Korea
}

\begin{abstract}
We report on the development of a high-resolution $(<1 \mathrm{GHz}$ at $1.0 \mathrm{THz})$, broadly-tunable $(\sim 0.5-3.0 \mathrm{THz})$ fiber MOPA-based terahertz spectrometer system. The flexibility and performance of this system will be demonstrated with gaseous (water vapor) and aqueous (water and solvated protein) samples with a view towards selectively and non-destructively probing functionally significant large-scale protein motions.
\end{abstract}

\section{Introduction and Background}

A narrow-bandwidth terahertz spectrometer with a tuning range limited to $0.4 \mathrm{THz}$ from a central wavelength has been previously demonstrated in our group by Malinowski et al. ${ }^{1}$. In this work we demonstrate our progress towards a significantly upgraded system tunable over a range in excess of $2.0 \mathrm{THz}$ (Fig.1).

Since protein folding dynamics take place on the picosecond timescale, vibrational modes tend to lie in the sub-2THz frequency range $\mathrm{e}^{2,3}$. Many recent studies have made extensive use of time-domain THz spectroscopy (TDS) ${ }^{4,5}$. The limited spectral resolution of the technique has generated a clear need for high-resolution $\mathrm{THz}$ sources, and recent development in this regard has been rapid and widespread ${ }^{6,7,8}$.

Our $\mathrm{THz}$ system has a similar dual fiber master oscillator power amplifiers (MOPA) chain design producing two pulsed beams of $2 \mathrm{~ns}$ duration at a repetition rate of $0.5 \mathrm{MHz}$. The maximum peak power per channel is $\sim 5 \mathrm{~kW}$ ( $5 \mathrm{~W}$ average power).

Both MOPA chains are designed to minimize spectral linewidth: one amplifier chain is based on a custom-built distributed feedback (DFB) laser operating at $\sim 1060 \mathrm{~nm}$, and the other is based on a Toptica DL pro 1040 tunable external cavity laser with a tuning range of $980-1075 \mathrm{~nm}$. The linewidth before amplification for both chains is of the order of 100 $\mathrm{kHz}$.

$\mathrm{THz}$ radiation is generated via difference frequency generation (DFG) in a (110) cleaved GaP crystal which is directed through a custom-built fluidic cell mounted in front of a silicon filter and pyroelectric $\mathrm{THz}$ detection system. A half-wave plate is located between the main system lens and the GaP crystal to achieve optimum polarization orientation with respect to the crystal orientation.

Both MOPA chains exhibit a flat gain response over the entire tuning range, with an optical signal-to-noise ratio (OSNR) of $\sim 30 \mathrm{~dB}$ (as measured at $0.1 \mathrm{~nm}$ resolution) which is particularly flat in the narrow range corresponding to the low frequency $(0.5-1.0 \mathrm{THz})$ region in the DFG process (Fig. 2). The estimated spectral linewidth of the MOPA output is $\sim 500$ $\mathrm{MHz}$ at $1060 \mathrm{~nm}$, corresponding to a $\sim 1 \mathrm{GHz}$ effective linewidth at $1.0 \mathrm{THz}$ with a typical peak THz power of $\sim 3 \mathrm{~mW}$.

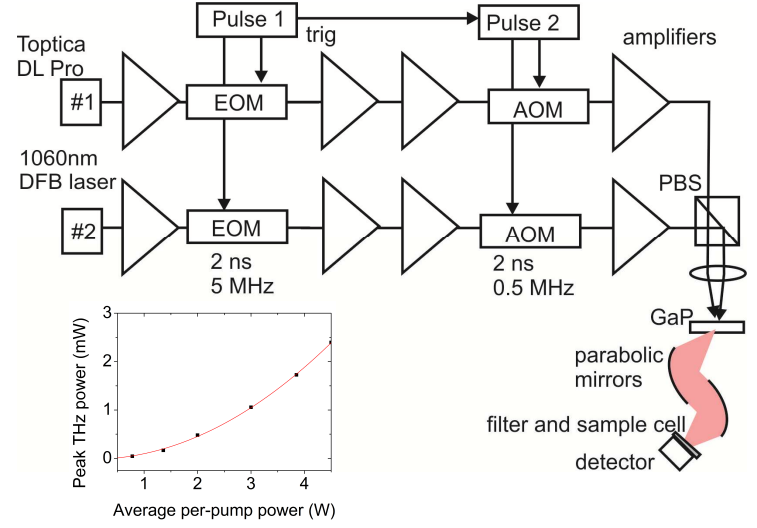

Fig. 1. ORC THz spectrometer design, showing dual fiber MOPA with PBS for beam combining and angle-tuning. Inset: THz power as a function of average incident pump power per MOPA chain.

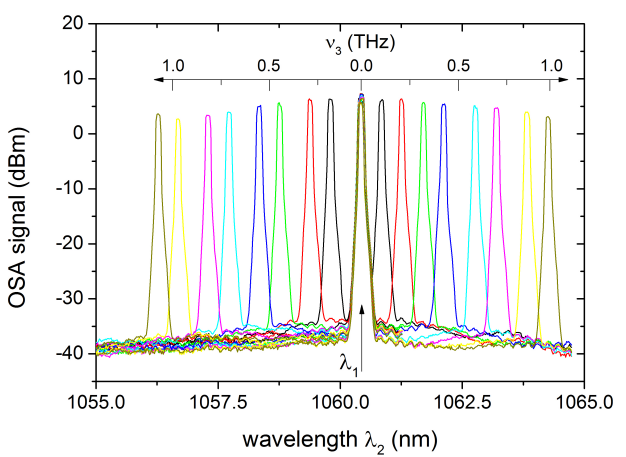

Fig. 2. MOPA output spectra. One MOPA chain is fixed at $\lambda_{1} \sim 1060 \mathrm{~nm}$, the other chain $\left(\lambda_{2}\right)$ is tunable. OSA resolution $=0.1 \mathrm{~nm}$. 
DFG of $\mathrm{THz}$ radiation can be achieved by passing both beams through the GaP crystal in either a collinear or (as in our case) in a non-collinear phase-matched configuration ${ }^{1}$. The tunable beam passes through the GaP perpendicular to the crystal face whilst the fixed wavelength $(1060 \mathrm{~nm})$ beam is passed through at an angle adjustable by translating the polarizing beam splitter (PBS) which is mounted on a micrometer-driven translation stage. By translating the PBS along the axis perpendicular to the GaP crystal face, the fixed wavelength beam's path is displaced perpendicularly to the axis of propagation and parallel to the optical table. The center of the GaP crystal sits at the shared focal point of both beams, translating the PBS corresponds to angle tuning the two beams. The Rayleigh ranges of the two beams are much longer than the thickness of the GaP crystal, ensuring efficient beam overlap.

To facilitate high-resolution vibrational spectroscopy of proteins the following upgrades have been implemented:

(i) Overall control of the $\mathrm{THz}$ spectrometer is consolidated in a single standard PC running Windows XP. An 8-port RS-232 card and USB system is used to communicate with the various computer-controllable components of the system.

(ii) The entire $\mathrm{THz}$ generation and detection sections are contained within a Perspex $\mathrm{N}_{2}$ purging shroud which features wedged antireflection coated entrance windows to eliminate reflections and etalon effects, and electrical feedthroughs.

(iii) Our compression-sealed flow cell for fluidic $\mathrm{THz}$ characterisation and protein manipulation is similar to a previous design ${ }^{910}$. Polytetrafluoroethylene (PTFE) film is compressed between two 5 mm thick PTFE discs by a 3D printed manifold, where a cutaway section of the film defines the microfluidic channels. The flow cell is mounted normal to the THz beam so the film thickness defines the path length of the biological sample. Holes are drilled in the lower manifold to connect capillaries to the microfluidic channels, allowing the delivery and disposal of multiple reagents as required for each protein experiment. .

(iv) A simplex lock-in amplifier type pyroelectric detector (Gentec T-Rad-USB) with an integration time of approximately $5 \mathrm{~s}$ is used to measure the throughput $\mathrm{THz}$ power. Using our narrow-band source, we aim to selectively probe and excite functionally relevant protein vibrational modes without denaturing the molecule. Such vibrational modes have previously only been non-specifically excited with a broadband source to cause denaturation ${ }^{11}$. Of particular interest are the lower frequency modes in the sub- $1.0 \mathrm{THz}$ region ${ }^{12}$, as these modes are most likely to be associated with functionally significant large-scale protein motions.

\section{Results}

To demonstrate the suitability of our fiber laser driven DFG based approach for precision spectroscopic measurements, we show early water vapor absorption data (Fig 3) comparing the DFG approach with conventional TDS with excellent agreement. We are soon to embark on novel studies in THz-induced protein dynamics, the confirmation of which would have significant implications for potential therapeutic applications. We hope to present our highresolution vapor and liquid-phase spectroscopic results in the very near future.

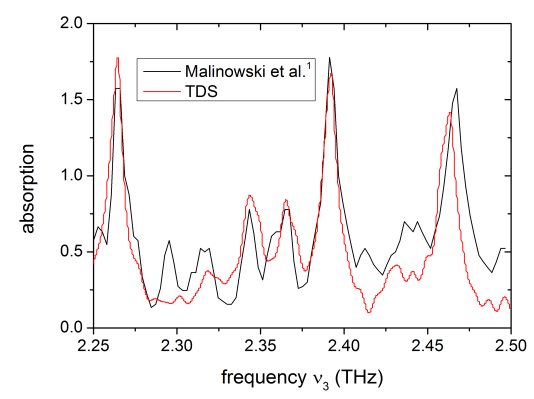

Fig. 3. Comparison of TDS and ORC system performance; water vapor absorption spectra in air are shown1.

\section{Outlook}

The terahertz spectrometer system described is capable of high-frequency-resolution excitation of protein folding, which will be exploited to selectively modulate the structure and function of proteins. Provision has been made for precise electronic control of exposure conditions in a flow-cell with transparent windows at $\mathrm{THz}$ and visible frequencies. Functional changes of proteins exposed to $\mathrm{THz}$ radiation will be evaluated using a well-characterized protein function test in free solution in the cell. Measurements on monomolecular films of protein immobilized at dielectric surfaces, equivalent to high sensitivity (multiple incidence) ellipsometry, have been demonstrated for calmodulin using commercial dualpolarization waveguide interferometry ${ }^{13}$ and similar complementary measurements here, under THz irradiation, would further elucidate observed protein function changes. While THz-induced changes in protein dynamics may not necessarily result in conformational changes, the demonstration of such an effect, especially when combined with assessments of protein function, would lend strong support for the protein electrodynamics hypothesis postulating functionally-specific interaction of $\mathrm{THz}$ radiation with proteins. 


\section{Acknowledgement}

This work is supported in part by Samsung Electronics Inc. under the Samsung Advanced Institute of Technology (SAIT) Global Research Outreach (GRO) Program.

\section{References}

[1] A. Malinowski, et al., "Fiber MOPA Based Tunable Source for Terahertz Spectroscopy," in Laser Physics Letters, Volume 9, No. 5, pages 350-354, 2012, DOI 10.7452/lapl.201110143.

[2] J. Xu, et al., "Probing the Collective Vibrational Dynamics of a Protein in Liquid Water by Terahertz Absorption Spectroscopy," in Protein Sci., Volume 5, No. 5, pages 1175-1181, 2006.

[3] C. Zhang, et al. "Broadened Far-Infrared Absorption Spectra for Hydrated and Dehydrated Myoglobin," in J. Phys. Chem. B, Volume 108, pages 10077-10082, 2004.

[4] T. Ding, et al., "Terahertz and Far Infrared Spectroscopy of Alanine-Rich Peptides having Variable Ellipticity," in Optics Express, Volume 18, Issue 26, pages 27431-27444, 2010.

[5] S. Q. Du, et al., "Vibrational Frequencies of Anti-Diabetic Drug Studied by Terahertz Time-Domain Spectroscopy," in Appl. Phys. Lett., Volume 100, pages $143702-1$ to $143702-4,2012$

[6] D. Xu, et al., "The Widely Tunable THz Generation in QPM-GaAs Crystal Pumped by a Near-Degenerate Dual-Wavelength KTP OPO at around

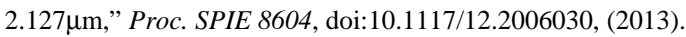

[7] K. Nawata, et al., "High-Power Tunable Terahertz-Wave Source Pumped by Dual-Wavelength Injection-Seeded Optical Parametric Generator," in 2012 Conference on Lasers and Electro-Optics (CLEO), Volume 49, Issue 3, pages 1-2, 2012.

[8] A. Lee, et al., "Frequency-Tunable THz Source Based on Stimulated Polariton Scatterig in Mg:LiNbO3," in IEEE Journal of Quantum Electronics, Volume 49, Issue 3, pages 357-364, 2013.

[9] D. J. Rowe, et al., "Microfluidic Device for Compositional Analysis of Solvent Systems at Microwave Frequencies," in Sensors and Actuators B: Chemical, Volume 169, pages 213-221, 2012.

[10] O. K. Castell, et al., "Liquid-Liquid Phase Separation: Characterisation of a Novel Device Capable of Separating Particles carrying Multiphase flows," in Lab Chip, Volume, No. 3, pages 388-396, 2009.

[11] T.E. Creighton, "Protein Folding," W.H. Freeman \& Co. Ltd., 1992.

[12] R.J. Falconer, A.G. Markelz, "Terahertz Spectroscopic Analysis of Peptides and Proteins," in J. Infrared Milli Teraherz Waves, No. 33, pages 973-988, 2012, DOI 10.1007/s10762-012-9915-9.

[13] K.E.D. Coan, et al., "Measurement and Differentiation of Ligand-Induced Calmodulin Conformations by Dual Polarization Interferometry," in Anal. Chem., Volume 84, pages 1586-1591, 2012. 